\title{
Helminth Infection in Freshwater Fishes of Bangladesh
}

\author{
Abu Tweb Abu AHmed \\ Department of Zoology, University of Dacca, Bangladesh
}

\begin{abstract}
Edible freshwater fishes common in central, northern and southern parts of Bangladesh were examined to record their helminth infection. A total of 78 taxa of helminths-1 monogenean, 28 digeneans, 8 caryophyllid cestodes, 24 nematodes and 17 acanthocephalans were recovered from 7 different organs of 37 species of freshwater fishes. Two species of trematodes (Ophiocorchis dasus GUPTA and Coitocaecum sp.), eight species of caryophyllids (Lytocestus indicus (MOGHE) WoodLAND, Lytocestus birmanicus LyNSDALE, Lytocestus parvulus FURTADo, Bovienia serialis (BOVIEN) FUHRMANn, Djombangia penetrans Bovien, Lucknowia sp., Capigentoides batrachii GuPTA and Pseudocaryophyllaeus indica GUPTA) and twelve species of acanthocephalans (Neoechineorhynchus topseyi PODDER, Neoechinorhynchus sp., Acanthosentis dattai PoDder, Acanthosentis indicus TrIPATHI, Acanthosentis tilapiae Baylis, Pallisentis allahabadii Agrawal, Pallisentis sp. 2, Pallisentis sp. 1 Devendrosentis garuai SAHAY et al., Hypoechinorhynchus sp., Heterosentis plotosi YAMAGUTI and Mehrarhynchus secundum TRIPATHI) are reported for the first time from Bangladesh.

Parasitic helminth incidence, infection intensity and distribution of the caryophyllid cestodes and acanthocephalans are also reported.
\end{abstract}

The helminth fauna of Indian subcontinent including Burma and Ceylon was first studied by SOUTHWELL (1913 \& 1930) and then by BAYLIS (1923, 1936 \& 1939). Later on, SRIVASTAVA (1936), GuPTA (1951, 1953, 1959, $1961 \& 1976)$ and TRIPATHI $(1954,1956 \& 1957)$ published a series of papers on fish helminths. In Bangladesh, BASHIRULLAH (1973) was the first to make a brief survey on the helminth fauna of fishes. He concentrated his studies mainly on the trematodes and nematodes. Recently, AHMED et al. (1976, 1977, 1978 $\& 1979$ ) have worked on the distribution, incidence, infection pattern and pathology of fish helminths in addition to their systematic studies.

This paper presents an uptodate checklist of the helminths so far isolated from different organs of freshwater fishes of Bangladesh with special attention to the parasitic incidence, infection intensity and distribution of caryophyllid cestodes and acanthocephalans.

\section{Materials and Methods}

The host fishes were collected at random from different fish ponds, ditches, rice fields, rivers and fish markets of central (Dacca and Tangail districts) northern (Mymensingh, Sylhet, Bogra and Rang- pur districts) and southern parts (Noakhali, Patuakhali and Barishal districts) of Bangladesh in different seasons of the year, mainly during summer and rainy seasons of 1976 to 1980 .

After external examination, body cavity of the host fishes (both live and those preserved in 5\% neutral formalin) was cut open and examined. Body cavity, mesenteries, musculature, alimentary canal, heart, liver, gall-bladder, spleen, kidney, swimbladder, gonads and urinary bladder were carefully removed to separate clean petri dishes containing $0.75 \%$ physiological saline solution. The organs were slit open carefully and examined under a stereobinocular microscope. The nemas were fixed in hot glacial acetic acid and the trematodes and caryophyllid cestodes in FAA. To keep the head organs intact caryophyllids and acanthocephalans were removed with special care by scraping deeply beneath them. Live acanthocephalans were kept in tap water until the osmotic pressure made them too weak to draw in the proboscis. They were then fixed in between the slides and preserved in $70 \%$ ethyl alcohol.

The worms, excepting the caryophyllids which were cleared in xylene, were cleared and temporarily mounted in lactophenol. Nematodes were stained in aniline blue, trematodes and cestodes in Semi- 
Table 1. Summary of the helminths recovered from freshwater fishes of Bangladesh

\begin{tabular}{|c|c|c|c|}
\hline Helminths & $\begin{array}{l}\text { Number } \\
\text { helminth } \\
\text { species }\end{array}$ & $\begin{array}{l}\text { Of host } \\
\text { species }\end{array}$ & $\begin{array}{c}\text { Organal distribution } \\
\text { (Number of helminth species) }\end{array}$ \\
\hline Monogenea & 1 & 1 & Gill (1) \\
\hline Digenea & 28 & 18 & $\begin{array}{l}\text { Body cavity (4), muscle \& liver (1), stomach (1), } \\
\text { stomach \& intestine ( } 3 \text {, intestine only (21), } \\
\text { swim bladder (2) and urinary bladder ( } 2) .\end{array}$ \\
\hline Caryophyllidea & 8 & 2 & $\begin{array}{l}\text { Stomach (2), anterior intestine (8) and posterior } \\
\text { intestine (5). }\end{array}$ \\
\hline Nematoda & 24 & 22 & $\begin{array}{l}\text { Body cavity (3), liver (1), stomach (6), stomach } \\
\& \text { intestine (1) and intestine only (23). }\end{array}$ \\
\hline Acanthocephala & 17 & 17 & $\begin{array}{l}\text { Liver, mesentery and intestine (2), stomach } \\
\text { and intestine (1) and intestine only (14). }\end{array}$ \\
\hline
\end{tabular}

cohn's carmine, while the acanthocephalans in borax carmine and hematoxylin and mounted in Canada balsam.

YamaGuTi's Systema Helminthum series (1958, $1959,1961,1963 \mathrm{a} \& 1963 \mathrm{~b}$ ) were followed for the study.

\section{Results and Discussion}

The results are shown in two parts:

I. Checklist of the helminths so far recovered from the freshwater fishes in Bangladesh.

II. Incidence, intensity of infection and distribution of caryophyllids and acanthocephalans.

I. A total of 78 species of parasitic helminths representing 47 genera and 26 families, have been isolated from different internal organs of 37 species of freshwater fishes (Table 1).

The following helminths are reported from Bangladesh for the first time: Ophiocorchis dasus Gupta, 1951, Coitocaecum sp., Lytocestus indicus (Moghe, 1925) Woodland, 1926, Lytocestus birmanicus LyNSDALE, 1956, Lytocestus parvulus Furtado, 1963, Bovienia serialis (Bovien, 1926) Fuhrmann, 1931, Djombangia penetrans Bovien, 1926, Lucknowia sp., Capingentoides batrachii GuPTA, 1961, Pseudocaryophyllaeus indica GUPTA, 1961, Neoechinorhynchus topseyi PODDER, 1937, Neoechinorhynchus sp. Acanthosentis dattai PODDER, 1938, Acanthosentis indicus TRIPATHI, 1956, Acanthosentis tilapiae BAYLIS, 1948, Pallisentis allahabadii Agrawal, 1958 Pallisentis sp. 1, Pallsentis sp. 2, Devendrosentis garuai SAHAY et al., 1972, Hypoechinorhynchus sp., Heterosentis plotosi YAMAGUTI, 1935 and Mehrarhynchus secundum
TRIPATHI, 1959.

Checklist of the helminths, their host fishes and site of infection are given in table 2:

It was observed that the trematodes and nematodes were common helminth parasites of freshwater fishes. The acanthocephalans were found to infect variety of fishes whereas the caryophyllids infected only siluroid catfishes, mainly Clarias which hosted seven species of the caryophyllids in addition to other helminths.

Same helminth parasite infected different host species and host organs e.g. Isoparorchis hypselobagri infected four different host organs of four host species. On the other hand, same host species harboured a number of helminth species in them e.g. Channa striatus and Channa punctatus carried nine species of helminths each, followed by Heteropneustes fossilis and Clarias batrachus which harboured eight helminth species each.

There is a relationship between the incidence and intensity of infection to age groups of fishes (Ahmed and Sanaullah, 1977). $1+$ age groups of fishes specially $H$. fossilis, $C$. batrachus and Channa spp. were heavily infested than those of 0 age groups. Food habit, too has similar relationship to the fish infection. For example, the extent of helminth infection of the herbivorous carps were insignificant comparing those with the omnivorous, voracious and predator snakeheads and catfishes.

II a. Out of the ten species of freshwater fishes examined, eight species of caryophyllid cestodes were found to occur only in catfishes-Clarias batrachus and Heteropneustes fossilis. Percentage of infection, infection intensity and organal distribution of the caryophyllids are given in Table 3 . 
Table 2. Checklist of the helminths, their host fishes and site of infection

\begin{tabular}{|c|c|c|}
\hline Helminth species & Hosts(s) & Organ(s) infected \\
\hline $\begin{array}{l}\text { Trematoda } \\
\text { Monogenea CARUS, } 1863 \\
\text { Dactylogyridae BACHOWSKY, } 1933\end{array}$ & & \\
\hline $\begin{array}{l}\text { Dactylogyrus glossogobii JAIN, } 1960 \\
\text { Digenea Van BENDEN, } 1858\end{array}$ & Glossogobius giuris & Gill \\
\hline Bucephalidae PoCHE, 1907 & & \\
\hline Bucephalus sp. & Mystus aor & Intestine \\
\hline $\begin{array}{l}\text { Neobucephalopsis aspinosiensis BASHIRULLAH \& } \\
\text { HAFIZUDDIN, } 1971\end{array}$ & Clupisoma garua & Intestine \\
\hline $\begin{array}{l}\text { Neobucephalopsis clupisomius BASHIRULLAH \& } \\
\text { HAFIZUDDIN, } 1973\end{array}$ & Clupisoma murius & Intestine \\
\hline $\begin{array}{l}\text { Neobucephalopsis sp. } \\
\text { Lecithodendriidae ODHNER, } 1910\end{array}$ & Clupisoma murius & Intestine \\
\hline $\begin{array}{l}\text { Pleurogenoides notopteri BASHIRULLAH \& } \\
\text { HAFIZUDDIN, } 1973 \\
\text { Opisthorchidae BRAUN, } 1901\end{array}$ & Notopterus notopterus & Intestine \\
\hline $\begin{array}{l}\text { Ophisthorchis sp. } \\
\text { Gorgoderidae Looss, } 1901\end{array}$ & Rita rita & Body cavity \\
\hline Phyllodistomum folium (OLTERS, 1816) BraUN, 1899 & $\begin{array}{l}\text { Glossogobius giuris } \\
\text { Channa striatus }\end{array}$ & $\begin{array}{l}\text { Body cavity } \\
\text { Urinary bladder }\end{array}$ \\
\hline Phyllodistomum yousufzai BashirullaH \& IslaM, 1970 & Rita rita & Swim bladder \\
\hline $\begin{array}{l}\text { Phyllodistomum sp. } \\
\text { Maseniidae YAMAGUTI, } 1954\end{array}$ & Channa marulius & Urinary bladder \\
\hline $\begin{array}{l}\text { Eumasenia } \text { sp. } \\
\text { Allocereadiidae STossıcH, } 1903\end{array}$ & Heteropneustes fossilis & Intestine \\
\hline Coitocaecum sp. & Mystus aor & Stomach \& intestine \\
\hline Opegaster beliyai PANDE, 1937 & Glossogobius giuris & Intestine \\
\hline Opegaster sp. & Glossogobius giuris & Intestine \\
\hline Eucredium daccai BashirullaH \& ElahI, 1972 & Channa punctatus & Intestine \\
\hline Crowicrocaecum channai BashirullaH \& ElaHI, 1972 & Channa marulius & Intestine \\
\hline Macrolecithus spp. & $\begin{array}{l}\text { Heteropneustes fossilis } \\
\text { Puntius sarana }\end{array}$ & $\begin{array}{l}\text { Intestine } \\
\text { Intestine }\end{array}$ \\
\hline Neopecoelina saharanpurensis GUPTA, 1955 & $\begin{array}{l}\text { Heteropneustes fossilis } \\
\text { Channa punctatus }\end{array}$ & $\begin{array}{l}\text { Intestine } \\
\text { Intestine }\end{array}$ \\
\hline $\begin{array}{l}\text { Neopecoelina sp. } \\
\text { Hemiuridae LUHE, } 1901\end{array}$ & Heteropneustes fossilis & Intestine \\
\hline $\begin{array}{l}\text { Lecithocladium daccai BASHIRULLAH \& } \\
\text { HAFIZUDDIN, } 1973\end{array}$ & Hilsa ilisha & Intestine \\
\hline Ophiocorchis dasus GUPTA, 1951 & $\begin{array}{l}\text { Glossogobius giuris } \\
\text { Channa punctatus }\end{array}$ & $\begin{array}{l}\text { Stomach \& intestine } \\
\text { Stomach \& intestine }\end{array}$ \\
\hline Genarchopsis lobata (SRIVASTAVA) 1933 & Channa gachua & Intestine \\
\hline Genarchopsis bengalensis BASHIRULLAH \& ELAHI, 1972 & Channa punctatus & Intestine \\
\hline $\begin{array}{l}\text { Genarchopsis ozaki BASHIRULlaH \& ElAHI, } 1972 \\
\text { Monorchidae ODHNER, } 1911\end{array}$ & Channa punctatus & Stomach \& intestine \\
\hline $\begin{array}{l}\text { Palaeorchis sp. } \\
\text { Fellodistomidae NicoLL, } 1913\end{array}$ & Puntius sarana & Stomach \\
\hline $\begin{array}{l}\text { Steringotrema sp. } \\
\text { Ophistholebetidae FuKUI, } 1929\end{array}$ & Puntius sophora & Intestine \\
\hline $\begin{array}{l}\text { Ophistholebes sp. } \\
\text { Isoparorchidae PocHe, } 1926\end{array}$ & Puntius sophore & Intestine \\
\hline Isoparorchis hypselobagri (BILLET, 1898) & Wallago attu & Swimbladder \\
\hline
\end{tabular}


Table 2. Continued

\begin{tabular}{|c|c|c|}
\hline Helminth species & Hosts(s) & Organ(s) infected \\
\hline & Channa punctatus & $\begin{array}{l}\text { Body cavity, liver, } \\
\& \text { muscle }\end{array}$ \\
\hline & Channa striatus & $\begin{array}{l}\text { Body cavity, liver } \\
\& \text { muscle }\end{array}$ \\
\hline & Channa marulius & $\begin{array}{l}\text { Body cavity, liver } \\
\& \text { muscle }\end{array}$ \\
\hline \multicolumn{3}{|l|}{ Cestoda } \\
\hline \multicolumn{3}{|l|}{ Caryophyllidea Ben. in Olsson, 1883} \\
\hline \multicolumn{3}{|l|}{ Caryophyllaeidae LEUKART, 1878} \\
\hline Lytocestus indicus (MoGHE, 1925) WoOdLAND, 1926 & Clarias batrachus & Stomach \\
\hline Lytocestus birmanicus LYNSDALE, 1956 & Clarias batrachus & Intestine \\
\hline Lytocestus parvulus FURTADO, 1963 & Clarias batrachus & Intestine \\
\hline Bovienia serialis (BovIEN, 1926) FUHRMANN 1931 & Clarias batrachus & Intestine \\
\hline Djombangia penetrans BovIEN, 1926 & Clarias batrachus & Stomach \\
\hline Lucknowia sp. & Heteropneustes fossilis & Intestine \\
\hline Capingentoides batrachii GUPTA, 1961 & Clarias batrachus & Intestine \\
\hline Pseudocaryophyllaeus indica GuPTA, 1961 & Clarias batrachus & Intestine \\
\hline \multicolumn{3}{|l|}{ Nematoda } \\
\hline \multicolumn{3}{|l|}{ Oxyuroidea WeINLAND, 1858} \\
\hline \multicolumn{3}{|l|}{ Oxyuridae COBBoLd, 1864} \\
\hline Cosmoxynemoides sp. & Colisa fasciata & Intestine \\
\hline \multicolumn{3}{|l|}{ Ascarididea YAMAGUTI, 1961} \\
\hline \multicolumn{3}{|l|}{ Ascarididae BLANCHARD, 1849} \\
\hline \multirow[t]{8}{*}{ Ascarididean larvae } & Mystus vittatus & Intestine \\
\hline & Bagarius bagarius & Intestine \\
\hline & Clarias batrachus & Intestine \\
\hline & Heteropneustes fossilis & Intestine \\
\hline & Ompok pabda & Intestine \\
\hline & Ompok bimaculatus & Intestine \\
\hline & Glossogobius giuris & Body cavity \\
\hline & Nandus nandus & Body cavity \\
\hline \multicolumn{3}{|l|}{ Quimperiidae BAYLIS, 1930} \\
\hline Buckleynema sp. & Mystus vittatus & Intestine \\
\hline \multicolumn{3}{|l|}{ Heterocheilidae RaILliet \& HenRy, 1951} \\
\hline Goezia sp. & Wallago attu & Intestine \\
\hline \multicolumn{3}{|l|}{ Spiruroidea DIESING, 1861} \\
\hline \multicolumn{3}{|l|}{ Camallanidae RaILliET and HeNRY, 1915} \\
\hline \multirow[t]{2}{*}{ Camallanus adamsia BASHIRULLAH, 1973} & Channa striatus & Intestine \\
\hline & Channa punctatus & Intestine \\
\hline Camallanus gibsonia BASHIRULLAH, 1973 & Channa striatus & Intestine \\
\hline \multirow[t]{3}{*}{ Camallanus spp. } & Puntius sophore & Intestine \\
\hline & Glossogobius giuris & Intestine \\
\hline & Channa marulius & Intestine \\
\hline Zeylanema anabantis (PEARSE, 1933) & Anabas testudineus & Intestine \\
\hline \multirow[t]{2}{*}{ Zeylanema pearsei $\mathrm{YEH}, 1960$} & Anabas testudineus & Intestine \\
\hline & Channa gachua & Intestine \\
\hline Zeylanema yehia BASHIRULLAH, 1973 & Channa gachua & Intestine \\
\hline Zeylanema tridensis BASHIRULLAH, 1973 & Channa gachua & Liver \\
\hline Zeylanema bidigitalis BASHIRULLAH, 1973 & Channa gachua & Intestine \\
\hline $\begin{array}{l}\text { Spirocamallanus inglisi BASHIRULLAH \& } \\
\text { HAFIZUDDIN, } 1973\end{array}$ & Notopterus notopterus & Intestine \\
\hline
\end{tabular}


Table 2. Continued

\begin{tabular}{|c|c|c|}
\hline Helminth species & Hosts(s) & Organ(s) infected \\
\hline Spirocamallanus viviparus (ALI, 1956) & Myctus vittatus & Intestine \\
\hline Spirocamallanus murius BASHIRULLAH \& HAFIZUDdIN & Clupisoma murius & Stomach \& intestine \\
\hline Spirocamallanus olsenia BASHIRULLAH, 1973 & Channa striatus & Intestine \\
\hline Spirocamallanus timmi BASHIRULLAH, 1973 & Mystus vittatus & Stomach \\
\hline \multirow[t]{3}{*}{ Spirocamallanus sp. } & Heteropneustes fossilis & Stomach \\
\hline & Mystus cavasius & Intestine \\
\hline & Clupisoma murius & Stomach \\
\hline Procamallanus cancilus BASHIRULLAH \& & Xenentodon cancila & Intesitine \\
\hline \multicolumn{3}{|l|}{ HAFIZUDDIN, 1973} \\
\hline Procamallanus daccai GuPTA, 1959 & Channa striatus & Intestine \\
\hline Procamallanus bangladeshi BASHIRULLAH \& & Heteropueustes fossilis & Stomach \\
\hline HAFIZUDDIN, 1973 & & \\
\hline \multicolumn{3}{|l|}{ Cucullanidae CoBBold, 1864} \\
\hline Cucullanellus sp. & Rita rita & Intestine \\
\hline Indocucullanus sp. & Mystus cavasius & Body cavity \\
\hline \multicolumn{3}{|l|}{ Gnathostomatidae LANE, 1923} \\
\hline \multirow[t]{3}{*}{ Gnathostoma spinigerum OWEN, 1836} & Mystus micropthalmus & Stomach \\
\hline & Wallago attu & Stomach \\
\hline & Channa striatus & Intestine \\
\hline \multicolumn{3}{|l|}{ Acanthocephala } \\
\hline \multicolumn{3}{|l|}{ Neoechinorhynchidea Southwell et MACFIE, 1925} \\
\hline \multicolumn{3}{|l|}{ Neoechinorhynchidae Van Cleave, 1919} \\
\hline Neoechinorhynchus topseyi PODDER, 1937 & Polynemus paradiseus & Intestine \\
\hline \multirow[t]{2}{*}{ Neoechinorhynchus sp. } & Cynoglossus lingua & Intestine \\
\hline & $\begin{array}{l}\text { Eleutheronema } \\
\text { tetradactylum }\end{array}$ & Intestine \\
\hline \multicolumn{3}{|l|}{ Acanthogyridae THAPAR, 1927} \\
\hline Acanthogyrus acanthogyrus THAPAR, 1927 & Catla catla & Intestine \\
\hline \multicolumn{3}{|l|}{ Quadrigyridae VAN Cleave, 1920} \\
\hline Acanthosentis dattai PODDER, 1938 & Barbus stigma & Intestine \\
\hline Acanthosentis indicus TRIPATHI, 1956 & Setipinna phasa & Intestine \\
\hline Acanthosentis tilapiae BAYLIS, 1948 & Channa striatus & Intestine \\
\hline Pallisentis nandai SARKAR, 1953 & Nandus nandus & $\begin{array}{l}\text { Liver, mesentery } \\
\& \text { intestine }\end{array}$ \\
\hline Pallisentis gaboes (MaCCallum, 1918) Van Cleave, 1928 & Channa striatus & Intestine \\
\hline Pallisentis allahabadii AGRAWAL, 1958 & Channa punctatus & $\begin{array}{l}\text { Liver, mesentery } \\
\& \text { intestine }\end{array}$ \\
\hline Pallisentis nagpurensis (BHALERAO, 1931) & Channa striatus & Intestine \\
\hline \multicolumn{3}{|l|}{ BAYLIS, 1933} \\
\hline Pallisentis sp. 1. & Glossogobius giuris & Intestine \\
\hline Pallisentis sp. 2. & Colisa fasciata & Intestine \\
\hline \multirow[t]{2}{*}{ Devendrosentis garuai SAHAY et al., 1972} & Clupisoma garua & Intestine \\
\hline & Silonia siloda & Stomach \\
\hline \multicolumn{3}{|l|}{ Echinorhynchidea Southwell et MACFIE, 1925} \\
\hline \multicolumn{3}{|l|}{ Echinorhynchidae COBBOLD, 1879} \\
\hline Hypoechinorhynchus sp. & Mystus gulio & Intestine \\
\hline Sachalinorhynchus sp. & Labeo rohita & Intestine \\
\hline \multicolumn{3}{|l|}{ Arhythmacantidae YAMAGUTI, 1935} \\
\hline Heterosentis plotosi Y AMAGUTI, 1935 & Plotosus canius & Intestine \\
\hline \multicolumn{3}{|l|}{ Rhadinorhynchidae Travassos, 1923} \\
\hline Mehrarhynchus secundum TRIPATHI, 1959 & Tachysurus gagora & Intestine \\
\hline
\end{tabular}


Table 3. Incidence, infection intensity and organal distribution of caryophyllid cestodes in two fish hosts

\begin{tabular}{|c|c|c|c|c|c|c|c|}
\hline \multirow[b]{2}{*}{ Hosts and Parasites } & \multicolumn{3}{|c|}{ Extent of infection } & \multirow{2}{*}{$\begin{array}{l}\text { Organs infected } \\
(\%)\end{array}$} & \multicolumn{3}{|c|}{ Infection intensity } \\
\hline & \multicolumn{3}{|c|}{$\begin{array}{l}\text { Number of fish } \% \\
\text { examined infected positive }\end{array}$} & & Range & $\begin{array}{l}\text { Mean } \\
\text { infected } \\
\text { fish }\end{array}$ & $\begin{array}{l}\text { for } \\
\text { total } \\
\text { fish }\end{array}$ \\
\hline \multicolumn{4}{|c|}{ Clarias batrachus (Linnaeus) } & Attached to the walls of: & & & \\
\hline $\begin{array}{l}\text { Lytocestus indicus } \\
\text { (MOGHE) WoODLAND }\end{array}$ & 36 & 14 & 38.9 & $\begin{array}{l}\text { Stomach }(3), \text { anterior }(89) \\
\& \text { posterior }(8) \text { intestine }\end{array}$ & $1-52$ & 14.2 & 5.5 \\
\hline $\begin{array}{l}\text { Lytocestus brimanicus } \\
\text { LYNSDALE }\end{array}$ & 12 & 1 & 8.4 & Anterior (100) intestine & $0-3$ & 3 & 0.3 \\
\hline $\begin{array}{l}\text { Lytocestus parvulus } \\
\text { FURTADO }\end{array}$ & 22 & 7 & 31.8 & $\begin{array}{l}\text { Anterior (82) \& posterior } \\
\text { (18) intestine }\end{array}$ & $1-18$ & 9.3 & 3 \\
\hline $\begin{array}{l}\text { Bovienia serialis } \\
\text { (BOVIEN) FUHRMANN }\end{array}$ & 28 & 12 & 42.9 & $\begin{array}{l}\text { Anterior (74) \& posterior } \\
\text { (26) intestine }\end{array}$ & $1-17$ & 7 & 3 \\
\hline $\begin{array}{l}\text { Djombangia penetrans } \\
\text { BovIEN }\end{array}$ & 35 & 20 & 57 & $\begin{array}{l}\text { Stomach }(7), \text { anterior }(89) \\
\& \text { posterior }(4) \text { intestine }\end{array}$ & $1-31$ & 10.3 & 5.9 \\
\hline $\begin{array}{l}\text { Capingentoides } \\
\text { batrachii GUPTA }\end{array}$ & 12 & 2 & 16.7 & Anterior intestine (100) & $1-5$ & 3 & 0.5 \\
\hline $\begin{array}{l}\text { Pseudocaryophyllaeus } \\
\text { indica GUPTA }\end{array}$ & 12 & 5 & 41.7 & $\begin{array}{l}\text { Anterior }(88) \& \text { posterior } \\
\text { (12) intestine }\end{array}$ & $1-17$ & 9.6 & 4 \\
\hline \multicolumn{8}{|c|}{ Heteropneustes fossilis (Bloch) } \\
\hline \multirow[t]{2}{*}{ Lucknowia sp. } & 32 & 1 & 3.2 & Anterior intestine (100) & $0-2$ & 2 & 0.1 \\
\hline & 189 & 62 & 32.8 & & $0-52$ & 10.3 & 3.4 \\
\hline
\end{tabular}

Geographical distribution of the cestodes, collected from Dacca, Tangail and Barisal districts are shown in Fig. 1.

The variablity of infection rate is likely to be correlated with the suitability of the habitat as a feeding ground of the distinctive hosts. Caryophy!lid infestation was heavy in fishes from undisturbed ponds and stagnant ditches where annelid hosts were abundant. The adult fishes were much infected.

Multiple infection of the caryophyllids was found in the intestine of $C$. batrachus. In addition, trematodes, nematodes and acanthocephalans were also found in the same habitat. The suitable temperature, rainfall and niche all over the country enhances excellent survival value and distribution of the helminths, specially the caryophyllids.

II b. 35 species of freshwater fishes including five estuarine fishes (from Khepupara, Patuakhali) were studied during May, 1979 to April, 1980 from Dacca and Patuakhali districts which are situated at a distance of about 125 miles. Of those, only 16 host species ( 66 specimens) were infected by 16 species of acanthocephalans representing 8 genera and 6 families ( 12 new record including 3 new species under 5 genera). 19 species of host fishes including Labeo rohita, were found uninfected and the percentage of host infection was 45.7. Sachalinorhynchus sp., earlier reported by us (Ahmed and

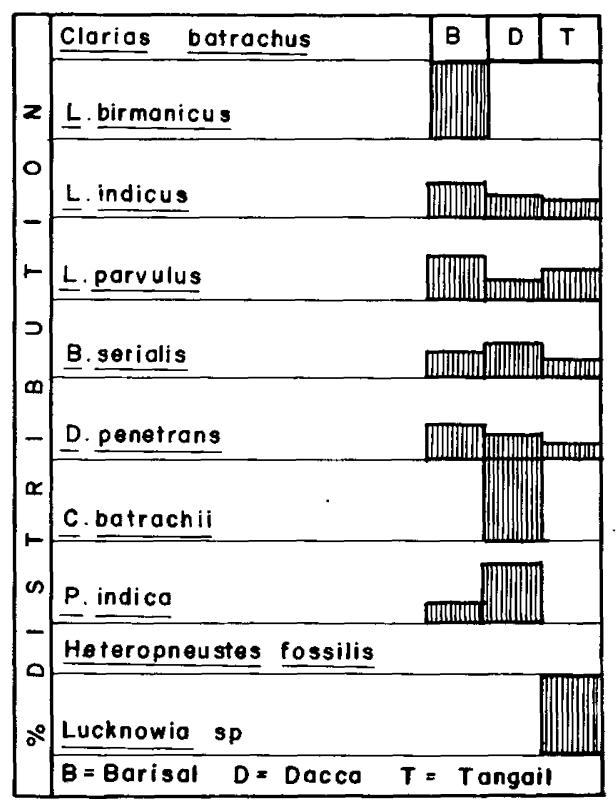

Fig. 1. Percentage distribution of caryophyllaeids to three differnt districts of Bangladesh. 
Table 4. Incidence, infection intensity and distribution of acanthocephalans in different host fishes collected from Patuakhali (P) and Dacca (D)

\begin{tabular}{|c|c|c|c|c|c|c|c|c|}
\hline \multirow{3}{*}{$\begin{array}{c}\text { Host fishes } \\
\text { (infected) }\end{array}$} & \multicolumn{4}{|c|}{ Occurrence } & \multirow[b]{2}{*}{ The acanthocephalans } & \multicolumn{3}{|c|}{ Infection intensity } \\
\hline & \multicolumn{3}{|c|}{$\begin{array}{l}\text { Number of fish } \\
\text { examined infected }\end{array}$} & \multirow{2}{*}{$\begin{array}{c}\% \\
\text { positive } \\
72.7\end{array}$} & & \multirow{2}{*}{$\begin{array}{l}\text { Range } \\
1-5\end{array}$} & $\begin{array}{l}\text { Mear } \\
\text { infected } \\
\text { fish }\end{array}$ & $\begin{array}{l}\text { n. for } \\
\text { total } \\
\text { fish }\end{array}$ \\
\hline & $\begin{array}{ll}\text { P } & 3 \\
\text { D } & 8\end{array}$ & D & 8 & & $\begin{array}{c}\text { Neoechinorhynchus } \\
\text { topseyi PODDER }\end{array}$ & & 2 & 1.5 \\
\hline Cynoglosuss lingua & P 3 & $\mathbf{P}$ & 1 & 33.3 & Neoechinorhynchus sp. & $0-1$ & 1 & 0.3 \\
\hline $\begin{array}{l}\text { Eleutheronema } \\
\text { tetradactylum }\end{array}$ & P 2 & $\mathbf{P}$ & 1 & 50 & Neoehcinorhynchus sp. & $0-1$ & 1 & 0.5 \\
\hline Catla catla & $\begin{array}{ll}\mathrm{P} & 3 \\
\mathrm{D} & 4\end{array}$ & D & 4 & 57.2 & $\begin{array}{l}\text { Acanthogyrus } \\
\quad \text { acanthogryus THAPAR }\end{array}$ & $2-12$ & 9.5 & 5.4 \\
\hline Barbus stigma & $\begin{array}{ll}\mathrm{P} & 7 \\
\mathrm{D} & 7\end{array}$ & $\mathrm{D}$ & 1 & 7.2 & $\begin{array}{l}\text { Acanthosentis dattai } \\
\text { PODDER }\end{array}$ & $0-1$ & 1 & 0.1 \\
\hline Setipinna phasa & $\begin{array}{lr}P & 13 \\
D & 5\end{array}$ & $\begin{array}{l}\mathrm{P} \\
\mathrm{D}\end{array}$ & $\begin{array}{l}8 \\
4\end{array}$ & 66.7 & $\begin{array}{l}\text { Acanthosentis indicus } \\
\text { TRIPATHI }\end{array}$ & $1-4$ & 1.7 & 1.1 \\
\hline Channa striatus & P 4 & $\mathrm{P}$ & 1 & 25 & $\begin{array}{l}\text { Acanthosentis tilapiäe } \\
\text { BAYLIS }\end{array}$ & $0-1$ & 1 & 0.3 \\
\hline Nandus nandus & $\begin{array}{l}\mathrm{P} \quad 6 \\
\mathrm{D} 18\end{array}$ & $\begin{array}{l}\mathrm{P} \\
\mathrm{D} 1\end{array}$ & $\begin{array}{r}3 \\
10\end{array}$ & 54.2 & $\begin{array}{l}\text { Pallisentis nandai } \\
\text { SARKAR }\end{array}$ & $2-8$ & 2.6 & 1.4 \\
\hline Channa striatus & $\begin{array}{ll}\mathrm{P} & 3 \\
\mathrm{D} & 3\end{array}$ & $\begin{array}{l}\mathrm{P} \\
\mathrm{D}\end{array}$ & $\begin{array}{l}2 \\
3\end{array}$ & 83.3 & $\begin{array}{l}\text { Pallisentis gaboes } \\
\text { (MaCCALlum) VAN } \\
\text { CleAve }\end{array}$ & $4-11$ & 6 & 5 \\
\hline Channa punctatus & $\begin{array}{ll}\mathrm{P} & 3 \\
\mathrm{D} & 5\end{array}$ & $\begin{array}{l}\mathrm{P} \\
\mathrm{D}\end{array}$ & $\begin{array}{l}3 \\
4\end{array}$ & 87.5 & $\begin{array}{l}\text { Pallisentis allahabadii } \\
\text { AGRAWAL }\end{array}$ & $1-3$ & 2.3 & 2 \\
\hline Channa striatus & $\begin{array}{ll}\mathrm{P} & 3 \\
\mathrm{D} & 2\end{array}$ & $\begin{array}{l}\mathbf{P} \\
\mathrm{D}\end{array}$ & $\begin{array}{l}2 \\
2\end{array}$ & 80 & $\begin{array}{l}\text { Pallisentis nagpurensis } \\
\text { (BHALERAO) Baylis }\end{array}$ & $2-5$ & 4.3 & 3.4 \\
\hline Glossogobius giuris & $\begin{array}{l}\mathrm{P} \\
\mathrm{D}\end{array}$ & $\mathrm{D}$ & 1 & 6.3 & Pallisentis sp. 1. & $0-1$ & 1 & 0.1 \\
\hline Colisa fasciata & $\begin{array}{ll}\mathrm{P} & 8 \\
\mathrm{D} & 4\end{array}$ & $\mathrm{D}$ & 1 & 8.4 & Pallisentis sp. 2. & $0-1$ & 1 & 0.1 \\
\hline Clupisoma garua & D 14 & D & 1 & 7.2 & $\begin{array}{l}\text { Devendrosentis garuai } \\
\text { SAHAY et al. }\end{array}$ & $0-1$ & 1 & 0.1 \\
\hline Silonia silondia & D 1 & $\mathrm{D}$ & 1 & 100 & $\begin{array}{l}\text { Devendrosentis garuai } \\
\text { SAHAY et al. }\end{array}$ & 1 & 1 & 1 \\
\hline Mystus gulio & P 9 & $\mathbf{P}$ & 1 & 11.1 & Hypoechinorhynchus sp. & $0-1$ & 1 & 0.1 \\
\hline Labeo rohita & $\begin{array}{lr}\mathrm{P} & 5 \\
\mathrm{D} & 12\end{array}$ & $\mathrm{D}$ & 4 & 33.3 & Sachalinorhynchus sp. & $0-13$ & 7.3 & 1.7 \\
\hline Plotosus canius & P 15 & $\mathbf{P}$ & 3 & 20 & $\begin{array}{l}\text { Heterosentis plotosi } \\
\text { YAMAGUTI }\end{array}$ & $1-3$ & 1.7 & 0.3 \\
\hline Tachysurus gagora & P 12 & $\mathbf{P}$ & 1 & 8.3 & $\begin{array}{l}\text { Mehrarhynchus } \\
\text { secundum TRIPATHI }\end{array}$ & $0-1$ & 1 & 0.1 \\
\hline & 193 & 70 & & 36.3 & & $0-13$ & 3.1 & 1.1 \\
\hline
\end{tabular}

Note: Pallisentis nandai infected liver, mesentery and intestine; one Devendrosentis garuai infected stomach while others were isolated from the intestine.

Begum, 1978) from the intestine of Labeo rohita in Dacca only is also included in the report. The extent of infection and intensity and distribution of the acanthocephalans are summarized in Table 4. The worms infested mainly the intestine of the hosts, though two of them were found, often in encysted condition, in the liver, mesentery and stomach.

Of the 17 species of acanthocephalans, 7 species viz. Neoechinorhynchus topseyi, Acanthogyrus acanthogyrus, Acanthosentis dattai, Pallisentis sp. 1, Pallisentis sp. 2, Devendrosentis garuai and Sachalinorhynchus sp. were restricted to Dacca whereas 5 species viz. Neoechinorhynchus sp., Acanthosentis tilapiae, Hypoechinorhynchus sp., Heterosentis plotosei and Mehrarhynchus secundum were exclusive to Patuakhali. The rest 5 species were distributed in both the areas. 
It should be mentioned here that the hosts of the five acanthocephalans, exclusive to Patuakhali, except $A$. tilapiae were generally not available in Dacca. According to YAMAGUTI (1961) they are the helminths of marine fishes. In the present study, they were however, collected from estuarine zone (Andermaniki river mouth). Silonia silonda was a new host record and Channa striatus harboured maximum species (3) of acanthocephalans while Neoechinorhynchus sp. and D. garuai occupied double hosts each. The size of the worms varied from $1.27 \mathrm{~mm}$ to $80 \mathrm{~mm}$ with a new record of $80 \mathrm{~mm}$ in one of the $N$. topseyi.

The acanthocephalans were abundant in summer and the intensity of host infection was higher in April, May and June. This was probably due to their breeding season then, as gravid females and juveniles were available in that season.

\section{References}

Ahmed, A. T. A. and M. Sanaullah (1976): Organal and percentage distribution of some metazoan parasites in Heteropneustes fossilis (BLOCH) and Clarias batrachus (L.). J. Asiat. Soc. Bangladesh, 2 (1), 7-15.

Ahmed, A. T. A. and M. Sanaullah (1977): Observations on the incidence and intensity of some helminths in different length groups of Heteropneustes fossilis (Bloch) and Clarias batrachus (L.). Dacca Univ. Stud., Part B, 15 (2), 91-98.

Ahmed, A. T. A. and R. Begum (1978): Studies on the distribution of some endoparasitic helminths in six freshwater fishes of Dacca and Barisal. Bangladesh J. Aquac., 1 (1), 52-60.

AHmed, A.T.A. and M. Sanaullah (1979): Pathological observations of the intestinal lesions induced by caryophyllid cestodes in Clarias batrachus (LINNAEUs) (Siluriformes: Clariidae). Fish Path., 14 (1), $1-7$.

Bashirullah, A. K. M. (1973): A brief survey of the helminth fauna of certain marine and freshwater fishes of Bangladesh. Bangladesh J. Zool., 1 (1), 63-81.

Baylis, H. A. (1923): Notes on Procamallanus spiralis BAYLIS, 1923 (Nematoda). Parasit., 15 (2), 137-138.

BAYLIS, H. A. (1936): Nematoda. Vol. 1 (Ascaroidea and Strongyloidea): Fauna of British India includ- ing Ceylon and Burma. p. 408.

BAYLIs, H. A. (1939): Nematoda. Vol. II (Filarioidea, Dioctophymoidea and Trichinelloidea). Fauna of British India including Ceylon and Burma. p. 274.

Gupta, S. P. (1951): Three new trematodes of the family Hemiuridae LUHE, 1901 from freshwater fishes of U. P. Ind. J. Helm., 3 (1), 41-54.

Gupta, S. P. (1953): Trematode parasites of freshwater fishes. Ind. J. Helm., 5 (1), 3-12.

Gupta, S. P. (1959): Nematode parasites of vertebrates of East Pakistan. III. Camallanidae from fishes, amphibia and reptiles. Can. J. Zool., 37, 771-779.

GuPTA, S. P. (1961): Caryophyllaeids (Cestoda) from freshwater fishes of India. Proc. Helm. Soc. Wash., 28, 38-50.

Gupta, S. P. and S. L. Verma (1976): On a new acanthocephala Acanthogyrus guptai n. sp. from intestine of freshwater fish, Labeo rohita (HAM.) from Lucknow. Riv. Parasitol., 37 (2-3), 143-150.

Southwell, T. (1913): Notes from the Bengal Fisheries Laboratory, Indian Museum. Rec. Ind. Mus., 9 (1), 79-103.

Southwell, T. (1930): Fauna of British India including Ceylon and Burma. Cestoda Vol. I \& II. Taylor \& Francis Publishers, Red Lion Court, Fleet Street, London. 391 pp.

Srivastava, H. D. (1936): New hemiurids (Trematoda) from Indian fishes. Pt. I. A. new parasite of the subfamily Prosorchinae Yamagutr, 1934. Proc. Nat. Acad. Sci. India, 6, 174-178.

TrIPATHI, Y. R. (1954): Studies on the parasites of Indian fishes. IV. Trematoda: Monogenea, Microcotylidae. Rec. Ind. Mus., 52, 231-248.

Tripathi, Y. R. (1956): Studies on the parasites of Indian fishes. V. Acanthocephala. Rec. Ind. Mus., 54 (1-2), 61-99.

TrIPATHI, Y.R. (1957): Monogenetic trematodes from fishes of India. J. Helminth., 9, 1-149.

Yamaguti, S. (1958): Systema Helminthum. The digenetic trematodes of vertebrates. Vol. 3. Pt. I \& II, Interscience Publishers, New York. 1575 pp.

Yamaguti, S. (1959): Systema Helminthum. The cestodes of vertebrates. Vol. 2. Pt I. The cestodes of fishes. $158 \mathrm{pp}$. Ibid.

Yamaguti, S. (1961): Systema Helminthum. Nematodes of vertebrate. Vol. 3. Pt. I \& II. Ibid. 1261 pp.

Yamagut, S. (1963a): Systema Helminthum. Monogenea and Aspidocotylea. Vol. 4. Ibid. 699 pp.

Yamaguri, S. (1963b): Systema Helminthum. Acanthocephala. Vol. 5. Ibid. 423 pp. 